FORMATION Formation emploi

Revue française de sciences sociales

120 | Octobre-Décembre 2012

Parcours de formation : la recomposition des ségrégations

\title{
Les retours aux études postsecondaires : une expression de l'éducation tout au long de la vie?
}

Returns to postsecondary education: an expression of lifelong Learning? Rückkehr zu Hochschulstudien: ein Ausdruck des lebenslangen Lernens? El retorno a los estudios post-secundarios: ¿una expresión de educación permanente?

Pierre Doray, Pierre Canisius Kamanzi, Benoît Laplante et María Constanza Street

\section{OpenEdition \\ Journals}

Édition électronique

URL : http://journals.openedition.org/formationemploi/3807

DOI : 10.4000/formationemploi.3807

ISSN : 2107-0946

Éditeur

La Documentation française

Édition imprimée

Date de publication : 31 octobre 2012

Pagination : 75-100

ISSN : 0759-6340

Référence électronique

Pierre Doray, Pierre Canisius Kamanzi, Benoît Laplante et María Constanza Street, « Les retours aux études postsecondaires : une expression de l'éducation tout au long de la vie? », Formation emploi [En ligne], 120 | Octobre-Décembre 2012, mis en ligne le 08 janvier 2015, consulté le 30 octobre 2020. URL : http://journals.openedition.org/formationemploi/3807 ; DOI : https://doi.org/10.4000/ formationemploi.3807

(ㄷ) Tous droits réservés 


\title{
Les retours aux études postsecondaires: une expression de l'éducation tout au long de la vie?
}

\author{
BENOÎT LAPLANTE \\ Professeur-chercheur au Centre Urbanisation Culture Société, Institut national de la recherche \\ scientifique, Montréal (Québec), CANADA \\ Pierre Doray \\ Chercheur au Centre interuniversitaire de recherche sur la science et la technologie et \\ professeur au département de sociologie de l'université du Québec à Montréal, CANADA \\ María Constanza Street \\ Doctorante au Centre Urbanisation Culture Société, Institut national de la recherche \\ scientifique, Montréal (Québec), CANADA \\ Pierre Canisius Kamanzi \\ Chercheur au Centre interuniversitaire de recherche sur la science et la technologie, \\ Département des fondements et pratiques en éducation, Faculté des sciences de l'éducation, \\ université Laval (Québec), CANADA
}

Résumé

Les retours aux études postsecondaires : une expression de l'éducation tout au long de la vie?

Cet article vise à comprendre le retour aux études des jeunes adultes canadiens après la sortie de l'enseignement postsecondaire avec ou sans diplôme. Utilisant les données longitudinales de l'Enquête auprès des jeunes canadiens en transition, on montre que le retour aux études est plus fréquent au cours des trois premiers trimestres qui suivent l'arrêt des études. Par ailleurs, le retour est influencé par les caractéristiques de la scolarité antérieure, certaines marques du passage à l'âge adulte (notamment le type d'occupation, le revenu et le fait de vivre en couple) confortant ainsi l'hypothèse de la persistance des effets des ségrégations sociales.

Mots clés : Formation tout au long de la vie, cheminement scolaire, reprise d'études, inégalité sociale, enseignement supérieur, Canada 
Abstract

Returns to postsecondary education: an expression of lifelong Learning?

This article aims to understand the return to school of Canadian young adults after leaving postsecondary education (PSE) with or without a diploma. Using longitudinal data from the Youth in Transition Survey, we show that the return to school is more common during the first three quarters after studies cessation. Return is also influenced by the characteristics of prior schooling, some traits of transition to adulthood (e.g. type of occupation, income and living in a couple), thus confirming the hypothesis of persistence effects of social segregation.

Keywords: Lifelong learning, school paths, returning to learning, social inequality, higher education, Canada

Journal of Economic Literature: I 29, J 24

Traduction : Auteurs

En 1972, l'UNESCO (Organisation des nations unies pour l'éducation, la science et la culture) publiait, sous la plume d'Edgar Faure, une proposition de transformation du rapport éducatif, mieux connue sous l'expression «d'éducation permanente " (Faure et al., 1972). Ce projet éducatif s'inscrit dans un mouvement d'institutionnalisation de l'éducation des adultes, très présent depuis la fin de la seconde guerre mondiale dans les pays développés. Ce projet vise un élargissement de l'accès à l'éducation et à la formation des individus, quel que soit leur âge. Autrement dit, l'éducation ne doit plus être réservée à l'enfance et à la jeunesse ; au contraire, elle doit être présente tout au long de la vie, que l'individu soit en emploi ou pas. Dès lors, les étapes de la vie ne s'emboîtent plus de manière linéaire selon le continuum famille-école-marché du travail-retraite. Près de vingt-cinq ans plus tard, l'UNESCO dépoussiérait le projet d'éducation permanente en mettant en exergue celui d'éducation et de formation tout au long de la vie (lifelong learning) (Delors, 1996). D'autres grandes organisations internationales comme l'OCDE (Organisation de coopération et de développement économiques) et l'Union européenne lui ont emboîté le pas, soulignant l'importance de maintenir les activités d'éducation et de formation à toutes les étapes de la vie des individus.

Ces deux projets éducatifs peuvent être lus comme une volonté de décloisonnement de l'accès aux ressources éducatives d'une société en fonction des âges. En ce sens, il serait aussi possible de les concevoir comme une volonté de lutter contre une certaine ségrégation éducative ou sociale en fonction de l'âge. De même, ces projets ont contribué à modifier le rapport entre l'école et la société, car ils s'inscrivent dans le prolongement des politiques de démocratisation de l'éducation (Coulter et Mandell, 2012). Un système scolaire qui vise l'égalité ne doit plus seulement faciliter les passages d'un ordre d'enseignement à un autre ou favoriser uniquement l'accroissement du taux de réussite scolaire. 
Il doit également permettre aux individus de retourner aux études à différents moments de leur vie pour diverses raisons.

Dès lors, il est intéressant de mieux comprendre les processus sociaux en œuvre dans les retours aux études et trois questions se posent : 1) Qu'est-ce qui pousse des individus qui ont quitté des études, avec ou sans diplôme, à y retourner ?2) Quels facteurs sociaux agissent comme des catalyseurs de ces retours ? 3) Dans quelle mesure peut-on considérer que l'éducation tout au long de la vie permet d'élargir la démocratisation scolaire?

Le présent article vise à contribuer à une meilleure compréhension des retours aux études. De nombreuses études ont déjà été consacrées à la participation à l'éducation des adultes. Elles ont souligné le rôle des caractéristiques individuelles, des milieux de travail et de l'organisation du champ de l'éducation des adultes sur la participation (Barton et Potevin, 1991 ; Doray et al., 2005 ; Desjardins et al., 2007 ; Knighton et al., 2009) ; d'autres se sont attachées à mieux appréhender les interruptions d'études et les retours chez les jeunes adultes (Cox et Ebbers, 2010 ; Crook, 1997; Lambert et al., 2004 ; Kiger et Johnson, 1997 ; Kwong et al., 1997), mais peu de recherches ont tenu compte des effets du temps sur cette question. L'originalité de notre apport est d'analyser les retours aux études grâce à une approche longitudinale. Cela permet de bien distinguer les effets de l'âge, du temps écoulé depuis la sortie et des différents facteurs sociaux, même si nous nous limiterons ici aux premières années de la vie active. Notre analyse s'appuie en effet sur des données provenant d'une enquête longitudinale auprès d'un échantillon de jeunes Canadiens qui avaient, en 1999 , entre 18 et 20 ans.

Dans un premier temps, nous reviendrons sur quelques repères théoriques portant sur les retours aux études. Nous poursuivrons par la description des différentes dimensions méthodologiques que nous avons utilisées pour produire les résultats que nous présentons dans la partie suivante. Nous proposons ensuite quelques pistes interprétatives, soulignant la variété des facteurs qui influencent ou modulent les retours aux études.

\section{Le retour aux études : un phénomène en augmentation}

La question des retours aux études s'inscrit dans la problématique plus large des parcours scolaires et plus globalement des parcours de vie, dans la mesure où les premiers sont en partie structurés par les expériences extrascolaires (Doray, 2011 ; Picard, Trottier et Doray, 2011). En d'autres termes, il s'agit de s'interroger sur les processus qui conduisent des individus à reprendre des études après une plus ou moins longue interruption. Ces processus peuvent être strictement d'ordre scolaire (le désir de terminer des études et d'obtenir un diplôme), tout comme ils peuvent être professionnels, par exemple aspirer à une promotion ou à une mobilité sociale et professionnelle. Ils peuvent s'inscrire dans des stratégies de remédiation scolaire ou de correction d'un parcours difficile en formation initiale (de 
Montlibert, 1977) ou s'expliquer par la désarticulation des différentes dimensions constitutives de l'entrée dans la vie adulte (Galland, 1996). Nombre d'adultes retournent aux études parce que les raisons qui les ont poussés à les interrompre n'existent plus, comme par exemple la maternité ou les difficultés financières (Raymond, 2008).

Les retours aux études sont de plus en plus nombreux. Lambert et al. (2004) constataient que $15 \%$ des étudiants âgés de 20 à 22 ans en 2001 avaient abandonné les études postsecondaires avant d'obtenir leur diplôme, mais que deux ans plus tard, la moitié d'entre eux était retournée à l'école. Une étude plus récente utilisant aussi les données de l'Enquête sur les jeunes en transition (EJET) permet d'établir que " environ $40 \%$ des jeunes adultes sont passés directement aux études postsecondaires après avoir obtenu leur diplôme d'études secondaires (les "non-stoppeurs"), alors que $30 \%$ avaient repoussé de plus de quatre mois leurs études postsecondaires après avoir obtenu leur diplôme d'études secondaires (les "stoppeurs») (Hango, 2007 et 2011). Ceci contribue à rompre la linéarité traditionnelle des étapes de vie, enfance-scolarité-travail-retraite, et à produire une désynchronisation des processus associés à l'entrée dans la vie adulte : départ des études et insertion sur le marché du travail, départ de la famille d'origine pour fonder sa propre famille, etc. (Gaudet, 2007 ; Gallant, 1996). Ce brouillage des frontières a conduit plusieurs chercheurs à parler d'une nouvelle étape de la vie évoquée sous différents termes : "adulescence " (Anatrella, 1988); "postadolescence» (Galland, 2001) et " adultes en émergence " (Arnett, 2004) ou "youthood" (Côté, 2006).

Par ailleurs, comme le soulignent Laplante et al.(2010), nombreux sont les adultes qui reprennent les études. Au Québec, par exemple, les étudiants de 25 ans et plus constituent près de la moitié ( $48 \%$ en 2006) des étudiants des universités. Un nombre élevé de jeunes adultes et d'adultes composent donc l'effectif étudiant.

Broekemer (2002) rapporte qu’aux États-Unis, les raisons les plus fréquentes du retour aux études sont l'acquisition d'une qualification pour augmenter les chances d'obtenir un meilleur emploi, l'apprentissage de nouvelles connaissances, le renforcement de l'estime de soi et l'obtention d'une promotion. Loin de s'exclure mutuellement, ces différents motifs peuvent se combiner. Smart et Pascarella (1987) ont montré que la décision de reprendre des études chez les jeunes adultes varie selon les caractéristiques de leur expérience scolaire antérieure, leurs conditions de vie actuelle et certaines caractéristiques sociodémographiques, particulièrement le sexe et l'état matrimonial. Sur le plan scolaire, les personnes qui ont quitté l'école malgré de bons résultats et une attitude positive vis-à-vis des cours ont tendance à reprendre rapidement les études. Il s'agit en général d'étudiants qui ont abandonné les études en raison de contraintes financières ou de responsabilités familiales. En revanche, pour ceux qui ont interrompu les études à la suite d'un parcours scolaire difficile marqué par le manque d'estime de soi, les troubles de comportement ou une attitude négative envers l'école, le retour est plus difficile (Davey et Jamieson, 2003). 
Le retour aux études est, par ailleurs, moins fréquent chez les individus qui jouissent de bonnes conditions de vie à la sortie de l'école. Obtenir rapidement un emploi qui correspond aux aspirations professionnelles réduit la probabilité du retour. À l'inverse, occuper un emploi qui correspond peu aux aspirations professionnelles accroît la frustration et incite à reprendre les études pour améliorer sa situation (Thomas, 2001). Selon Marcus (1986), la reprise d'études est moins fréquente chez ceux qui ont eu la chance d'obtenir un bon emploi que chez ceux qui ont décroché des emplois offrant des conditions de travail moins intéressantes, notamment en matière de salaire. Comme l'observe Dayton (2005), certains reprennent des études par choix - la satisfaction personnelle ou la motivation professionnelle -, d'autres le font sous contrainte parce qu'ils estiment qu'une formation supplémentaire constitue le meilleur moyen de résoudre les problèmes d'instabilité sociale et financière auxquels ils sont confrontés.

Enfin, la probabilité de reprise d'études varie selon les caractéristiques sociodémographiques : le statut socio-économique des parents, la situation conjugale, l'origine ethnique, le lieu de résidence (rural ou urbain), etc. L'étude de Kwong, Mok et Kwong (1997) montre qu'aux États-Unis, le retour aux études est moins fréquent chez les jeunes adultes issus d'une famille à faible revenu, chez ceux qui ont des enfants à charge et chez les membres des minorités ethniques, en particulier les Noirs et les Hispanophones.

Pour Kiger et Johnson (1997) et Sftor et Turner (2002), les adultes qui reprennent des études, particulièrement ceux qui ont des enfants à charge, sont souvent vulnérables et ont besoin d'une aide financière suffisante pour consacrer moins de temps au travail salarié et s'investir davantage dans leurs études. Cox et Ebbers (2010) ont mis en exergue la vulnérabilité des femmes par rapport aux hommes.

\section{L'influence des facteurs individuels et du temps écoulé depuis l'arrêt des études}

Le retour aux études a été peu étudié au Canada (Tomkowicz et Busnik, 2003 ; Lambert et al., 2004 ; Hango, 2007 et 2011). On ne connaît pas suffisamment le phénomène pour formuler des hypothèses fortes a priori. Nous avons plutôt formulé deux questions génériques :

- Quels sont les facteurs qui influencent le retour aux études postsecondaires?

- À quel moment le retour aux études postsecondaires est-il plus fréquent?

Nous nous concentrons sur les effets des facteurs individuels et familiaux. Examiner les effets des facteurs structuraux, notamment ceux du système d'enseignement postsecondaire qui, au Canada, varie d'une province à l'autre, exigerait une étude spécifique que l'état actuel des connaissances ne permet pas de réaliser (Doray et al., 2009 ; Fisher et al., 2007 ; 2009). Nous nous concentrons sur les facteurs individuels qui semblent être les 
plus significatifs : le niveau du programme d'études précédent, les caractéristiques sociodémographiques - le capital scolaire des parents, la situation conjugale, le fait d'avoir eu des enfants ou non - et les conditions de vie - le fait d'avoir un emploi ou pas, le nombre moyen d'heures travaillées par semaine, le régime de travail, le niveau de compétence de l'emploi et le niveau du revenu selon le régime de travail et la catégorie de travailleur. On doit cependant admettre que la probabilité de reprendre des études, si on ne l'a pas déjà fait, varie en fonction du temps écoulé depuis l'arrêt : au-delà de l'effet des facteurs qui semblent les plus prégnants, la probabilité de retourner aux études n'est vraisemblablement pas la même au cours de chacun des trimestres qui suivent la fin d'un cheminement scolaire ou l'interruption des études. En outre, il est difficile d'affirmer a priori que les effets des différents facteurs qui favorisent ou pénalisent le retour aux études ne changent pas en fonction du temps. Pour ajouter à la complexité, nous supposons que les effets des facteurs varient selon qu'on a quitté les études après avoir achevé son programme d'études ou en l'abandonnant.

En pratique, la probabilité de reprendre les études dépend donc du temps écoulé depuis la fin des études et d'un certain nombre de facteurs dont les effets dépendent d'abord à la fois de la manière dont on a mis fin à ses études, en achevant un programme ou en l'abandonnant. Pour mieux comprendre ce phénomène, l'analyse doit être réalisée à l'aune d'un modèle statistique qui tient compte de cette complexité.

\section{Une enquête pour étudier les transitions significatives}

\subsection{L'enquête et l'échantillon : un suivi des jeunes sur sept ans}

Nous utilisons les données de l'Enquête auprès des jeunes en transition (EJET), enquête à passages répétés réalisée conjointement par Statistique Canada et par le ministère des Ressources humaines et du Développement des compétences du Canada. Les questionnaires de l'EJET recueillent de l'information sur plusieurs des éléments importants de la vie des jeunes, dont la plupart des épisodes d'études ou d'emploi. Cette information permet d'étudier plusieurs des transitions importantes qui peuvent survenir à ce moment de la vie : la fin des études secondaires, le début des études postsecondaires, l'obtention du premier emploi, le départ du foyer parental, etc. Ces questionnaires recueillent également de l'information sur les facteurs qui peuvent influencer ces transitions, certains de nature " objective ", notamment le contexte familial et les expériences scolaires antérieures, et d'autres de nature "subjective ", comme les aspirations et les attentes (Statistique Canada, 2007, p. 83). 
L'EJET a débuté en 1999 et le premier passage - le "cycle 1 »-a servi à recueillir de l'information sur l'année 1999. Les questionnaires utilisés au cours des passages suivants ont servi à recueillir de l'information sur des périodes de deux ans. Le " cycle 2 » a ainsi recueilli de l'information sur les années 2000 et 2001, le " cycle 3 " sur les années 2002 et 2003 et le « cycle 4 » sur les années 2004 et 2005. L'EJET a donc permis d'observer la vie des enquêtés pendant sept ans.

La cohorte que nous utilisons est formée de jeunes nés entre 1979 et 1981 inclusivement ; ils étaient âgés de 18 à 20 ans au 31 décembre $1999^{1}$. Les analyses portent sur les enquêtés qui résident dans une des dix provinces du Canada et qui ont répondu aux quatre cycles de l'enquête. Les analyses portent sur le retour aux études après avoir obtenu le premier diplôme post-secondaire ou après avoir abandonné un programme d'études postsecondaires sans en avoir obtenu le diplôme. La période d'observation couvre les années 1999 à 2005. Notre sous-échantillon comprend 5613 personnes dont 3314 ont interrompu les études après avoir obtenu le diplôme d'études postsecondaires et 2299 qui ont abandonné avant l'obtention du diplôme.

\subsection{Modéliser les processus de retour aux études}

Nous étudions les processus qui régissent le retour aux études grâce à un modèle de risque (ou de probabilité instantanée) à temps discret ; on trouvera une introduction à l'usage des modèles de risque en sciences sociales par exemple dans Box-Steffensmeier et Bradford (2004).

Selon la nature du programme auquel s'inscrivent les enquêtés qui reprennent des études, nous distinguons deux formes de retour : le retour dans un programme universitaire ou pré-universitaire, et le retour dans un programme professionnel.

Le calendrier scolaire canadien et la manière dont l'EJET recueille l'information ne permettent pas de considérer qu'une personne qui n'est pas inscrite dans un cheminement depuis au moins deux trimestres a vraiment arrêté ses études. Ainsi, nous n'étudions pas la reprise d'études à partir du moment de la fin des études, mais bien à partir du début du troisième trimestre qui suit la fin des études.

Une personne qui a complété ou abandonné ses études depuis deux trimestres est, dans le langage statistique de l'analyse longitudinale, "à risque » d'entreprendre soit un cheminement universitaire ou pré-universitaire, soit un cheminement postsecondaire non universitaire professionnel. La personne qui ne suit plus des études depuis plus de deux trimestres est donc soumise à deux risques concurrents, celui du retour aux études dans un cheminement universitaire ou pré-universitaire et celui du retour aux études dans un cheminement professionnel. En pratique, ceci signifie qu’à tout moment cette per-

1. Le plan de sondage de l'EJET exclut les populations qui habitent les trois «territoires » - c'est-àdire les portions du territoire canadien peu peuplées qui n'ont pas le statut de province -, les réserves indiennes, les bases des Forces canadiennes et certaines régions éloignées. 
sonne peut occuper trois statuts différents : 1) ne pas suivre d'études, 2) avoir entrepris un cheminement universitaire ou pré-universitaire ou 3) avoir entrepris un cheminement professionnel. On doit alors estimer, pour la population à risque, les effets des variables indépendantes sur la probabilité, à chaque trimestre, de suivre un programme universitaire ou pré-universitaire, et les effets des mêmes variables sur la probabilité, à chaque trimestre, de suivre un programme postsecondaire non universitaire. Utiliser la régression logistique multinomiale est la manière la plus simple de le faire. La régression logistique multinomiale permet d'estimer les effets d'une ou plusieurs variables indépendantes sur une variable dépendante qualitative qui possède plus de deux modalités ; nous l'utilisons pour étudier deux processus concurrents, d'une manière analogue à celle qu'Allison (1982) et Kiefer (1990) ont popularisée pour étudier un seul processus au moyen de la régression logistique.

Nous estimons le risque de base et les effets des facteurs à chaque trimestre, qui peuvent donc varier d'un trimestre à l'autre. Cela nous permet d'examiner le calendrier du retour aux études. Nous estimons le risque de base et l'effet de chacun de ces facteurs nets de l'effet du sexe, de l'âge et de la région de résidence.

\section{Encadré 1}

\section{Le modèle}

Nous utilisons un modèle à temps discret que nous estimons au moyen de la régression logistique multinomiale. On peut résumer notre modèle en écrivant :

$$
\begin{gathered}
h^{R}(t \mid \hat{\mathbf{x}}, z)=h_{0}^{R}(t) \exp \left(\mathbf{x}^{R}\right) \\
\operatorname{avec} \hat{\mathbf{a}}^{R}=g(t \mid z),
\end{gathered}
$$

où $h^{R}(t)$ représente le taux instantané (ou probabilité instantanée ou risque instantané) de retourner aux études dans un programme universitaire ou professionnel - selon la valeur que l'on donne à $R-, h_{0}{ }^{R}(t)$ représente le taux « de base » du retour aux études dans un programme universitaire ou professionnel au cours d'un trimestre donné, $t$ représente le nombre des trimestres écoulés depuis la fin des études, $\mathbf{x}$ représente le vecteur des facteurs qui augmentent ou réduisent le taux, $\boldsymbol{\beta}^{R}$ représente le vecteur des effets de ces facteurs sur la probabilité du retour aux études dans un programme universitaire ou dans un programme professionnel selon la valeur de $R$, et $z$ représente le fait d'avoir mis fin à ses études en terminant un programme ou en l'abandonnant. Les effets $\left(\boldsymbol{\beta}^{R}\right)$ des facteurs $(\mathbf{x})$ varient en fonction du temps écoulé depuis qu'on a arrêté ses études $(t)$ et de la manière dont on y a mis fin ( $z$ ). On trouvera le détail de ce modèle dans Laplante et al. (2010). 


\section{Les
résultats}

Nous abordons la description des résultats en examinant l'effet du temps sur les retours aux études, leur fréquence selon le nombre de trimestres écoulés depuis le départ précédent des études.

Ensuite, nous nous attardons sur l'effet de différentes variables sociodémographiques et scolaires.

\subsection{Un retour aux études plus fréquent pour les non-diplômés}

Les figures 1 et 2 montrent la proportion des diplômés et des non-diplômés qui sont retournés aux études selon le nombre de trimestres écoulés depuis la sortie, en distinguant le niveau du programme postsecondaire qu'ils ont entrepris au cours de ce trimestre.

Figure 1

\section{Les sorties avec diplôme}

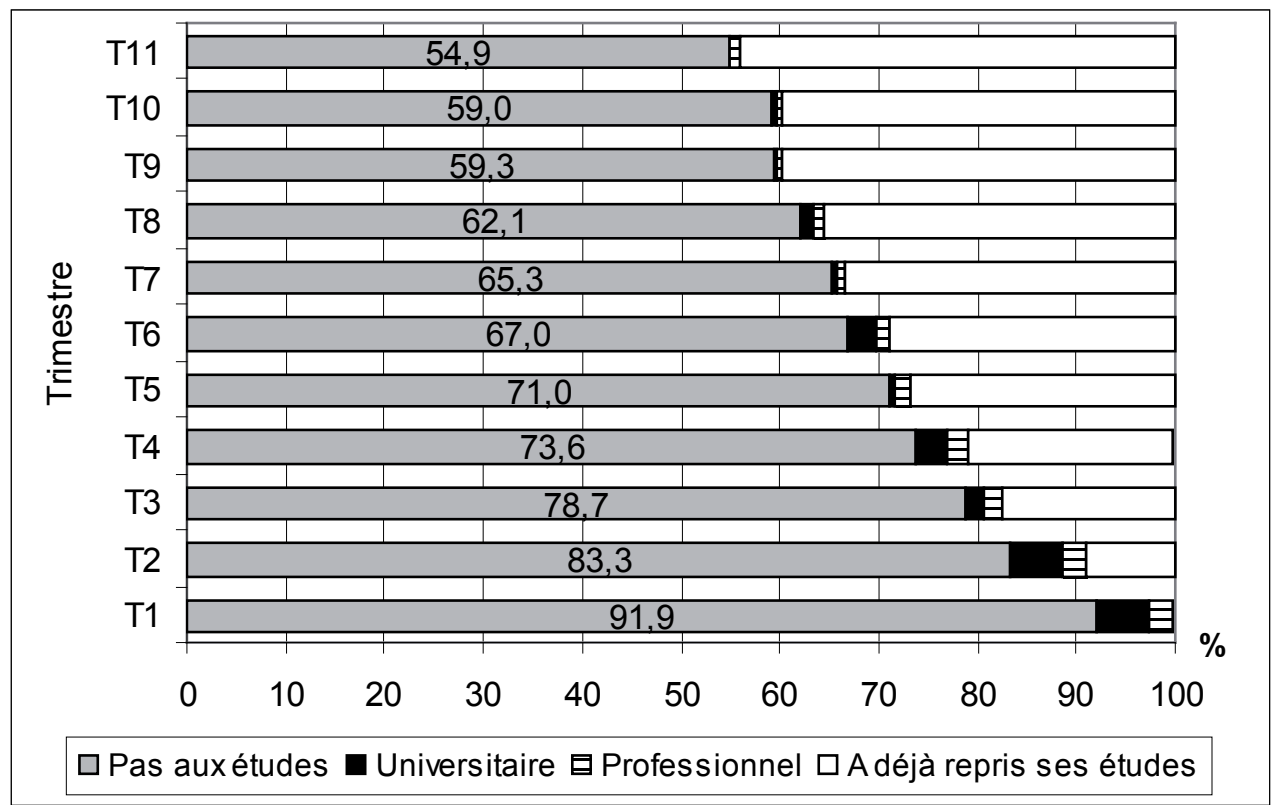


Figure 2

Les sorties sans diplôme

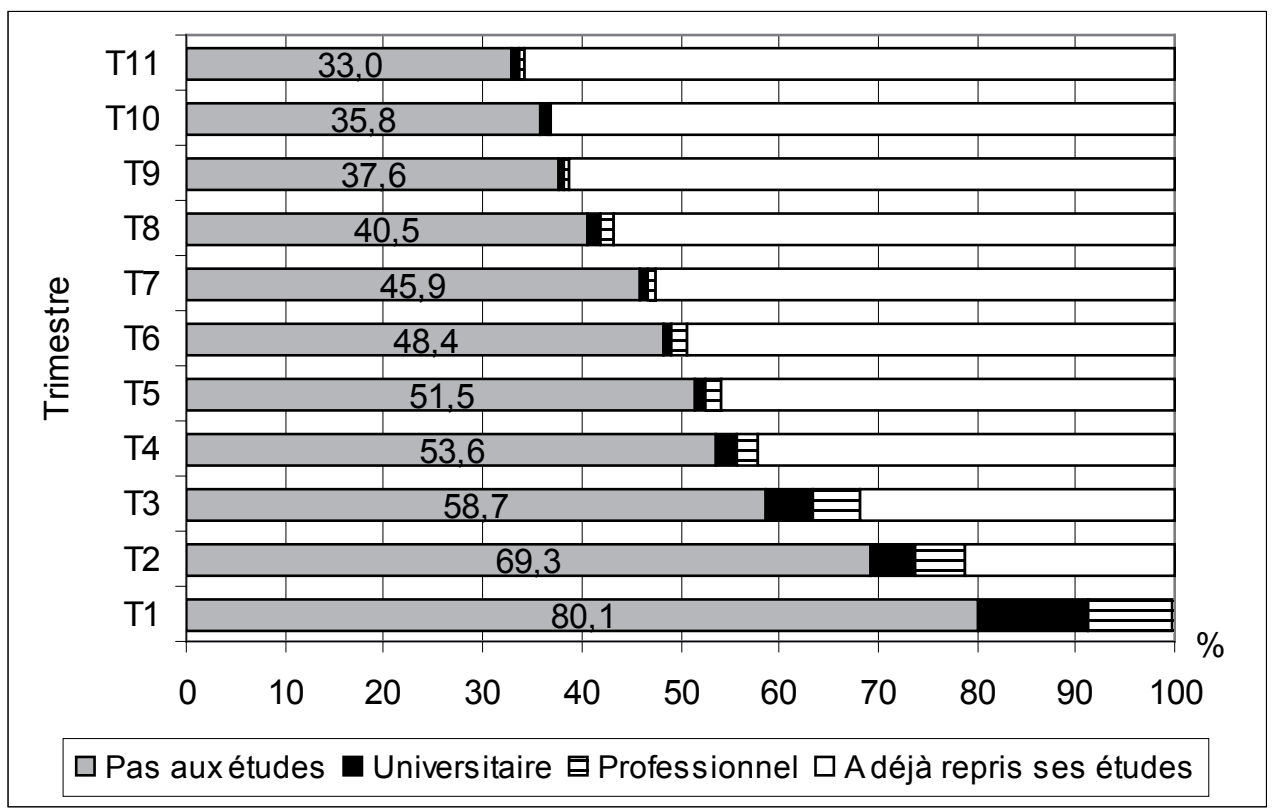

Note : Les figures 1 et 2 indiquent le mouvement de retour aux études entre le premier trimestre (T1) et le onzième trimestre (T11) après l'arrêt des études. Ainsi, à la figure 2, la barre T2 indique qu'au deuxième trimestre, la propor-tion de ceux qui ont effectué un retour aux études est légèrement supérieure à $20 \%$ parmi ceux qui avaient arrêté les études avant l'obtention d'un diplôme. La barre T6 de la même figure montre qu'au sixième trimestre, cette propor-tion avait atteint plus de $50 \%$.

Source : auteurs.

Ainsi, le retour aux études est plus fréquent parmi ceux qui n’ont pas achevé leurs études que chez les diplômés. Parmi les personnes qui ont quitté le postsecondaire depuis deux trimestres (T1 et T2), 16,7 \% (100 - 83,3) des diplômés et 30,7 \% $(100$ - 69,3) des nondiplômés ont repris des études postsecondaires au cours des deux premiers trimestres qui ont suivi. Au cinquième trimestre $(\mathrm{T} 5)$ - c'est-à-dire sept trimestres après la sortie -, la proportion est d'environ $30 \%$ et $50 \%$ respectivement. L'écart entre les deux groupes se maintient jusqu'à la fin de la période d'observation. Onze trimestres (T11) plus tard, soit six ans après la sortie, la proportion de jeunes qui sont retournés aux études atteint $45 \%$ chez les diplômés et 66 \% chez les sortants sans diplôme.

Quant au niveau du programme choisi, les diplômés sont davantage retournés dans un programme universitaire, surtout quand le retour a été plus précoce, alors que les nondiplômés reprennent plus fréquemment les études dans un programme professionnel que les diplômés. 


\subsection{Le taux de retour aux études décroît avec l'âge}

Les figures 3 à $\mathbf{8}$ montrent les taux de retour dans un programme universitaire ou professionnel par âge en contrôlant le sexe, la région de résidence et le niveau du programme précédent.

Figure 3

Sortie avec diplôme - Premier trimestre dans la population à risque

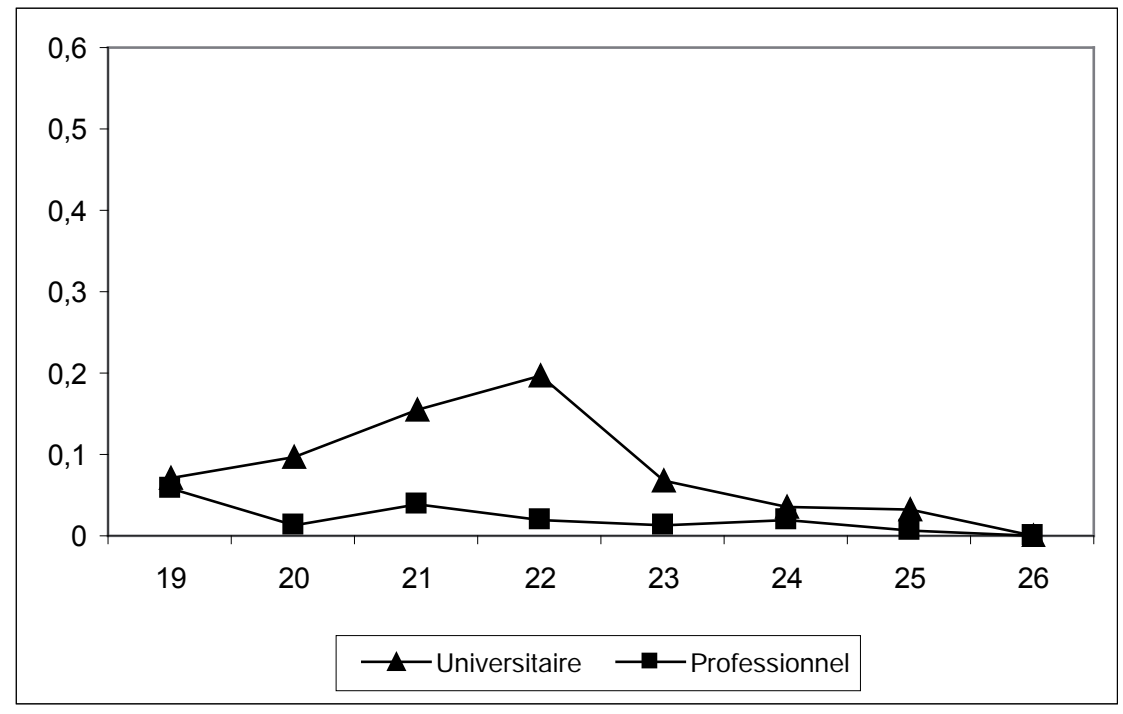

Figure 4

Sortie sans diplôme - Premier trimestre dans la population à risque

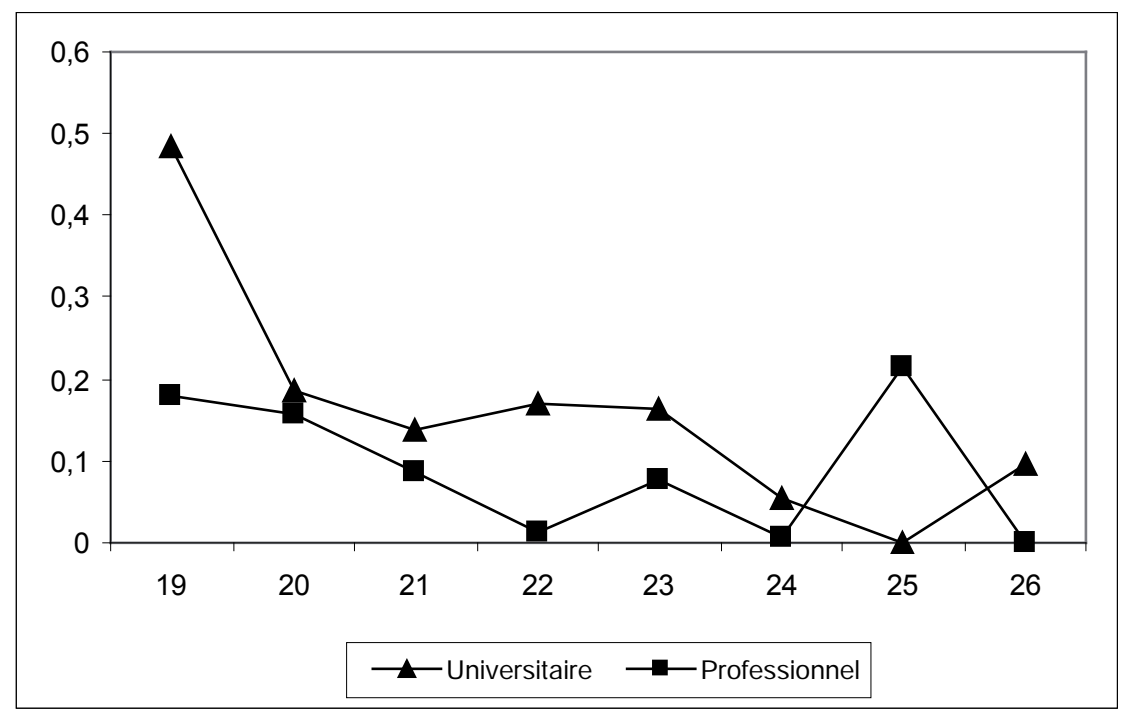


Figure 5

Sortie avec diplôme - Troisième trimestre dans la population à risque

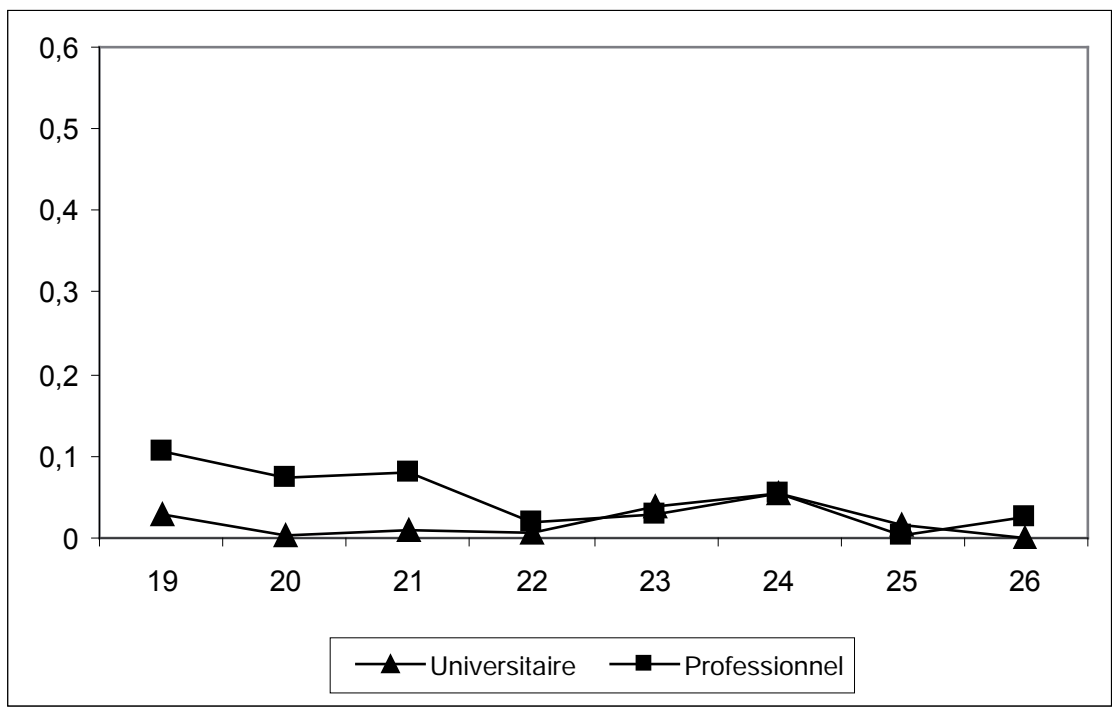

Figure 6

Sortie sans diplôme - Troisième trimestre dans la population à risque

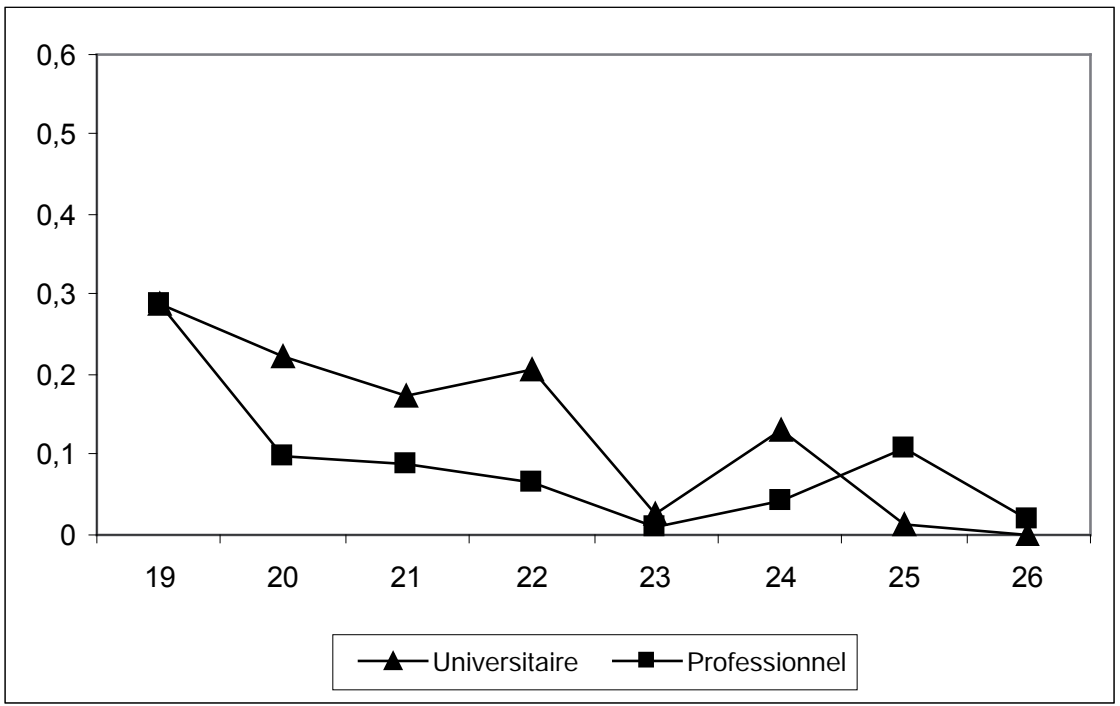


Figure 7

Sortie avec diplôme - Sixième trimestre dans la population à risque

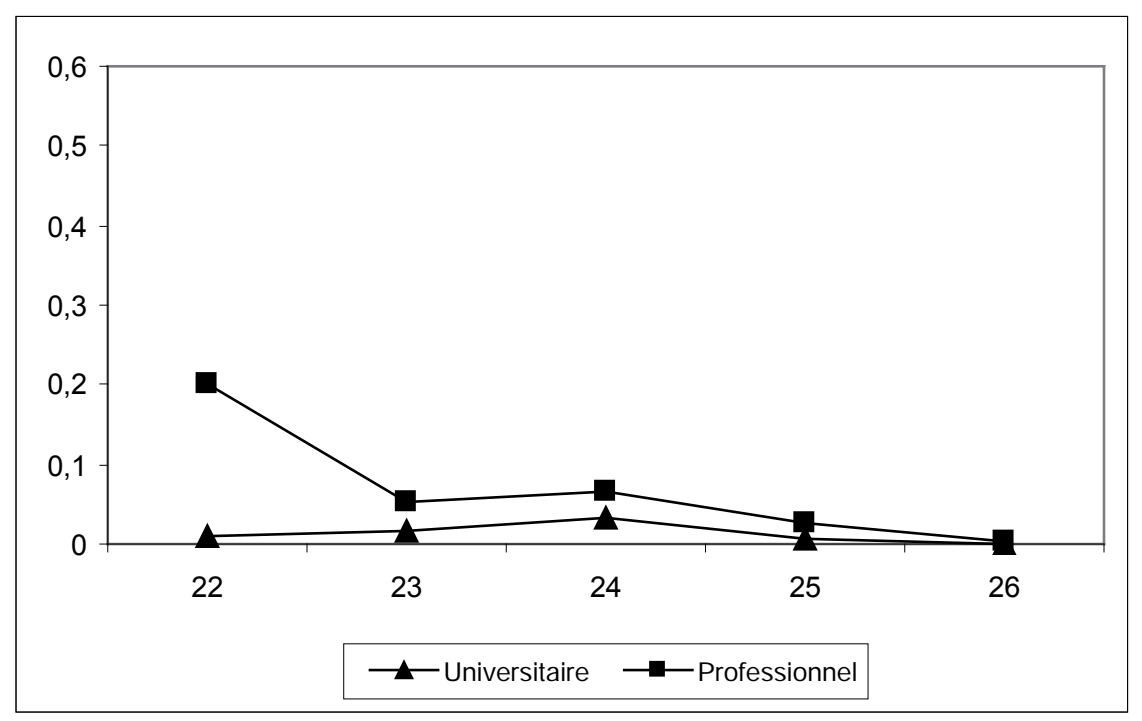

Figure 8

Sortie sans diplôme - Sixième trimestre dans la population à risque

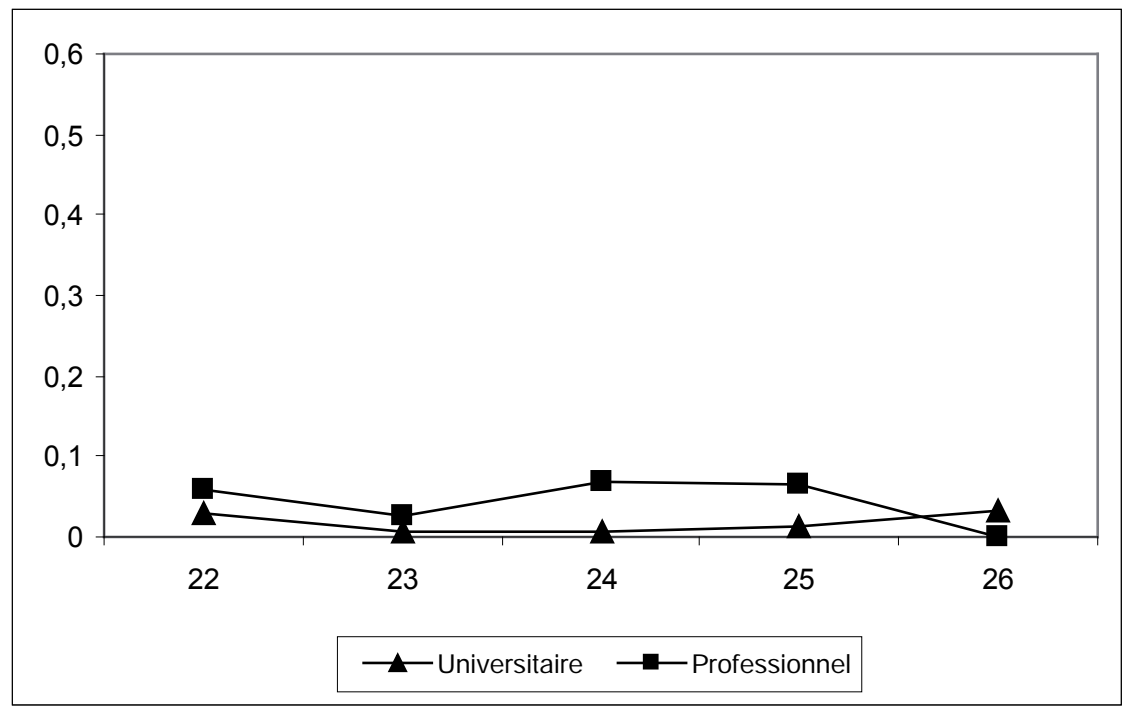

Note : Les figures 3 à 8 indiquent le risque de retour aux études selon l'âge, le temps écoulé après l'arrêt des études selon que les répondants sont sortis avec ou sans diplôme.

Source : auteurs. 
Le modèle permet de montrer que l'effet de l'âge sur la probabilité de reprendre les études au cours d'un trimestre donné si on ne l'a pas encore fait varie d'un trimestre à l'autre. Le modèle prévoit également que chaque variable indépendante peut avoir un effet différent, d'un trimestre à l'autre, sur la probabilité de retourner aux études. L'effet de l'âge ne se réduit pas à un seul coefficient : dans notre contexte, cette variable prend huit valeurs différentes et chacune de ces valeurs exprime un effet différent. Pour réduire la masse d'informations à présenter et à commenter, nous nous limitons à présenter l'effet de l'âge au cours du premier, du troisième et du sixième trimestre, pendant lesquels les individus sont susceptibles de reprendre les études.

Au premier trimestre, pour les diplômés, le taux de retour dans un programme universitaire augmente de 19 à 22 ans, puis il diminue. Pour les non-diplômés, le taux de retour dans un programme universitaire est très élevé avant 20 ans, assez élevé de 20 à 23 ans et assez faible chez les individus plus âgés. Pour les diplômés, le taux de retour dans un programme professionnel est faible à tous les âges, mais légèrement plus élevé avant 20 ans. Pour les non-diplômés, le taux de retour dans un programme professionnel est relativement élevé avant 20 ans, diminue de 20 à 22 ans et semble assez faible aux âges plus élevés. Cela pourrait s'expliquer, entre autres, par le fait que, plus un jeune adulte vieillit, plus il s'engage dans les responsabilités associées à l'âge adulte, parfois difficiles à concilier avec les études. Nous y reviendrons plus loin.

Au troisième trimestre, pour les diplômés, le taux de retour dans un programme universitaire est faible et ne semble pas varier en fonction de l'âge. Pour les non-diplômés, le taux de retour dans un programme universitaire est relativement élevé à 19 ans, il est plus faible, mais encore élevé entre 20 et 22 ans, et plus faible par la suite. Pour les diplômés, le taux de retour dans un programme professionnel est faible à tous les âges. Pour les non-diplômés, le taux de retour dans un programme professionnel est élevé à 19 ans, relativement faible mais encore appréciable entre 20 et 22 ans, et faible aux âges plus élevés.

Au sixième trimestre (T6), pour les diplômés, le taux de retour dans un programme universitaire est faible et ne semble pas varier en fonction de l'âge. Il en est de même pour les non-diplômés. Le taux de retour dans un programme professionnel est élevé pour les diplômés à 19 ans et faible à tous les autres âges; ce taux est faible à tous les âges pour tous les non-diplômés.

En résumé, le taux de retour aux études décroît avec l'âge. L'effet de l'âge, lorsqu'il existe, semble s'atténuer avec le temps écoulé depuis la fin des études. Une exception notable cependant : le taux de retour aux études universitaires augmente entre 19 et 22 ans au cours du premier trimestre au cours duquel les individus sont susceptibles de reprendre les études après les avoir interrompues. 


\subsection{Pas de différence significative entre hommes et femmes}

On ne trouve aucune différence statistiquement significative entre les hommes et les femmes, même au seuil de 0,1 , lorsqu'on tient compte de l'âge, de la région de résidence et du niveau du programme antérieur. Il est possible qu'il existe des différences entre les sexes dans la manière dont l'âge, la région de résidence ou le niveau du programme antérieur affectent le risque du retour aux études. Les effectifs ne permettent pas d'estimer de telles relations conditionnelles, pas plus qu'ils ne permettraient d'estimer des équations séparément pour les hommes et les femmes.

\subsection{Peu de retour vers un programme universitaire pour les détenteurs d'un diplôme professionnel}

Le tableau 1 (page suivante) montre les effets nets du niveau du programme précédent en prenant le niveau universitaire comme modalité de référence de la variable indépendante. Les étudiants qui ont obtenu un diplôme de niveau professionnel ont un risque plus faible de retourner aux études dans un programme universitaire ou menant à l'université : durant les cinq premiers trimestres, ce risque ne représente que $15 \%$ à $24 \%{ }^{2}$ du risque des diplômés universitaires. Le même comportement s'observe parmi les décrocheurs durant les trois premiers trimestres après la sortie. Cependant, il n'y a pas de différence significative entre les étudiants qui ont achevé un cheminement de niveau universitaire ou l'ont abandonné et ceux qui ont achevé un programme pré-universitaire ou l'ont abandonné. En conclusion, le retour dans un programme universitaire est moins fréquent parmi ceux qui ont suivi des études postsecondaires dans un programme menant au marché du travail, alors que le retour dans un programme professionnel ne semble pas être associé au niveau du programme précédent.

2. Pour les personnes qui ont quitté les études avec obtention du diplôme, les coefficients significatifs oscillent entre 0,153 et 0,234 , ce qui s'interprète comme un risque qui fluctue entre $15 \%$ et $24 \%$. Il est possible d'interpréter les autres informations du tableau 1 de la même manière. 
Tableau 1

Risque de retour aux études dans un programme universitaire ou professionnel à chaque trimestre selon le niveau du programme précédent et le type de sortie du postsecondaire. Effets nets en contrôlant l'âge, le sexe et la région de résidence

\begin{tabular}{|c|c|c|c|c|}
\hline \multirow{3}{*}{\begin{tabular}{|} 
Niveau du \\
programme antérieur
\end{tabular}} & \multirow{2}{*}{\multicolumn{2}{|c|}{ Sortie avec diplôme }} & \multirow{2}{*}{\multicolumn{2}{|c|}{ Sortie sans diplôme }} \\
\hline & & & & \\
\hline & $\begin{array}{c}\text { Retour dans } \\
\text { un programme } \\
\text { universitaire ou } \\
\text { préuniversitaire }\end{array}$ & $\begin{array}{l}\text { Retour dans } \\
\text { un programme } \\
\text { professionnel }\end{array}$ & $\begin{array}{c}\text { Retour dans } \\
\text { un programme } \\
\text { universitaire ou } \\
\text { préuniversitaire }\end{array}$ & $\begin{array}{l}\text { Retour dans un } \\
\text { pro-gramme } \\
\text { professionnel }\end{array}$ \\
\hline & $\%$ & $\%$ & $\%$ & $\%$ \\
\hline \multicolumn{5}{|l|}{ Trimestre 1} \\
\hline Préuniversitaire & 85,1 & 156,7 & $-30,8$ & $-29,3$ \\
\hline Professionnel & $-84,7^{*}$ & 43,5 & $-85,5^{*}$ & 34,5 \\
\hline Non classé & $-7,6$ & $-3,2$ & $-0,4$ & -100 \\
\hline \multicolumn{5}{|l|}{ Trimestre 2} \\
\hline Préuniversitaire & $-22,9$ & $-36,5$ & $-47,7$ & 18,9 \\
\hline Professionnel & $-76,6^{*}$ & $-34,3$ & $-76,3^{*}$ & 19,3 \\
\hline Non classé & $-81,9$ & -100 & -100 & 56,4 \\
\hline \multicolumn{5}{|l|}{ Trimestre 3} \\
\hline Préuniversitaire & 320,9 & 137,4 & $-71,4$ & $-31,2$ \\
\hline Professionnel & $-52,1$ & 46,4 & $-79,5^{*}$ & 43,2 \\
\hline Non classé & -100 & -100 & -100 & -100 \\
\hline \multicolumn{5}{|l|}{ Trimestre 4} \\
\hline Préuniversitaire & $-69,6$ & 237,5 & 44,2 & $-81,8$ \\
\hline Professionnel & $-79^{*}$ & 28,5 & $-38,1$ & 3,9 \\
\hline Non classé & $-92,6$ & -100 & -100 & -100 \\
\hline \multicolumn{5}{|l|}{ Trimestre 5} \\
\hline Préuniversitaire & $-99,9^{*}$ & 310,4 & $-14,5$ & 195,6 \\
\hline Professionnel & $-80,6^{*}$ & 100,7 & $-42,2$ & 82,2 \\
\hline Non classé & -100 & 132,9 & -100 & -100 \\
\hline \multicolumn{5}{|l|}{ Trimestre 6} \\
\hline Préuniversitaire & $-43,5$ & $-95,1$ & 127,1 & $-83,7$ \\
\hline Professionnel & $-3,5$ & $-71,2$ & $-73,8$ & -24 \\
\hline Non classé & -100 & -100 & 131,9 & 60,2 \\
\hline \multicolumn{5}{|l|}{ Trimestre 7} \\
\hline Préuniversitaire & 649,9 & 77,4 & -97 & $-5,7$ \\
\hline Professionnel & 163,4 & $-20,6$ & $-78,6$ & 23,5 \\
\hline Non classé & -100 & -100 & -100 & 990,8 \\
\hline
\end{tabular}

1) * : $p<0,05 ; 2$ ) Catégorie de référence : programme universitaire.

3) Les rapports de risque s'interprètent comme les rapports des côtes dans une analyse de régression logistique. Ils indiquent la variation du risque de changement d'état associé à l'événement étudié. Ainsi, le coefficient 1,81 (première colonne, première ligne) indique que, par rapport à la catégorie de référence (programme universitaire), le risque de retour aux études dans un programme préuniversitaire s'accroît de 85 \% (1,851 - 1). De même, le coefficient 0,153 (première colonne, deuxième ligne) indique que le risque de retour dans un programme professionnel plutôt que dans un programme universitaire diminue de $85 \%(0,153-1)$. Source : auteurs. 


\section{Encadré 2}

L'éducation postsecondaire au Canada

Il existe, au Canada, treize systèmes éducatifs, un par province ou territoire, chacun ayant des spécificités institutionnelles. À des fins de comparaison, il est nécessaire de gommer les différences régionales. Pour ce faire, nous pouvons distinguer deux programmes génériques : les études techniques ou professionnelles et les études universitaires. En sortant de l'enseignement secondaire général, les élèves peuvent choisir de poursuivre leurs études dans un collège dans des programmes professionnels ou aller à l'université. C'est cette distinction que nous reprenons dans notre analyse. En outre, au Québec, le système est différent dans la mesure où, sortant du secondaire, les élèves désireux de poursuivre des études universitaires doivent d'abord s'inscrire dans un programme pré-universitaire dans les collèges d'enseignement général et professionnel (CEGEP). En pratique, on ne peut pas gommer parfaitement les différences institutionnelles entre les provinces et les territoires. Le pré-universitaire mène normalement à l'université et est, par ailleurs, équivalent à l'université dans les autres provinces (*).

$\left(^{*}\right)$ : Dans nos analyses, nous maintenons la différence entre le pré-universitaire et l'universitaire comme cheminement scolaire d'origine - c'est-à-dire comme variable indépendante -, mais assimilons le préuniversitaire à l'universitaire comme cheminement de destination - variable dépendante.

\subsection{Les facteurs modulant les retours aux études}

Trois autres facteurs favorisent le retour aux études dans un programme universitaire ou menant à l'université lorsqu’on détient déjà un diplôme : résider au Québec, avoir des parents qui ont suivi des études universitaires et occuper un emploi salarié de 9 à 16 heures par semaine ${ }^{3}$. Quatre facteurs pénalisent ce retour : avoir terminé des études postsecondaires de niveau professionnel, vivre en couple, avoir un travail salarié permanent ou non permanent et travailler à temps plein.

Les facteurs qui réduisent la probabilité du retour aux études universitaires des diplômés sont des marques du passage à la vie adulte. Dans la mesure où l'on admet que les études concourent à se préparer à vivre comme un adulte de manière indépendante, le temps des études paraît révolu lorsqu'on a un conjoint et un emploi à temps plein.

Un seul facteur favorise le retour aux études dans un programme professionnel lorsqu'on détient déjà un diplôme : résider dans les Prairies ${ }^{4}$. Quatre facteurs compromettent ce retour : avoir un travail salarié permanent, travailler à temps plein, occuper un poste semi-professionnel ou intermédiaire et avoir un revenu moyen ou élevé. Comme ceux

3. Rapporter les résultats détaillés de l'estimation des effets des autres facteurs exigerait d'ajouter huit tableaux semblables au tableau 1. La chose semble difficile à justifier et nous nous limitons ici à les résumer. Pour le détail des résultats, nous renvoyons le lecteur à la note de recherche que les auteurs ont récemment publiée à ce sujet (Laplante et al., 2010).

4. La région des Prairies regroupe trois provinces du Canada : l'Alberta, la Saskatchewan et le Manitoba. 
qui réduisent le retour aux études universitaires, les facteurs qui réduisent la probabilité du retour aux études professionnelles des diplômés sont des marques du passage à la vie adulte. Ils sont cependant plus nettement liés à l'intégration économique et au succès professionnel.

Un seul facteur favorise le retour aux études dans un programme universitaire ou menant à l'université lorsqu'on a abandonné ses études postsecondaires : avoir des parents qui ont suivi des études universitaires. Sept facteurs compromettent ce retour : avoir suivi un programme professionnel, résider dans les Prairies, vivre en couple, avoir un travail salarié permanent, travailler à temps plein, occuper un poste semi-professionnel ou intermédiaire, et avoir un revenu moyen.

Finalement, le retour aux études dans un programme professionnel lorsqu'on a abandonné ses études postsecondaires est influencé par un seul facteur : avoir des parents qui ont suivi des études postsecondaires. Six facteurs compromettent ce retour : résider dans les Prairies, vivre en couple, avoir un travail salarié permanent, travailler à temps plein, occuper un poste semi-professionnel ou intermédiaire, avoir un revenu moyen.

\section{Pistes interprétatives : des effets qui se combinent}

Nous nous intéressons au calendrier du retour aux études postsecondaires et aux facteurs qui accélèrent ou ralentissent le retour aux études postsecondaires, plus spécialement l'influence du niveau du programme d'études précédent, des caractéristiques sociodémographiques et des conditions de vie. Nous admettons que le processus qui régit le retour aux études peut varier selon qu'on a mis fin à ses études après avoir obtenu le diplôme ou au contraire en les abandonnant.

Deux constatations s'imposent d'emblée : le retour aux études est fréquent au Canada, et le risque de retourner aux études varie selon le temps écoulé depuis la fin des études. La plupart des étudiants qui ont interrompu les études ou ont cessé d'étudier après l'obtention d'un diplôme se réinscrivent durant les trois premiers trimestres au cours desquels nous les considérons à risque de retourner aux études, c'est-à-dire entre le troisième et le cinquième trimestre qui suivent le trimestre de la dernière inscription. Ainsi, la première conclusion qui se dégage de l'étude est que le retour est plus probable dans les deux trimestres qui suivent l'arrêt des études. En d'autres termes, le risque de retourner aux études diminue avec le temps écoulé depuis la fin des études.

D’un côté, le retour aux études est influencé par la scolarité antérieure. Il est plus fréquent chez les jeunes qui sont sortis sans avoir obtenu le diplôme que chez les diplômés. Dès lors, on peut faire l'hypothèse que nombre de ceux qui reprennent les études postsecondaires sont motivés par le souci d'acquérir un diplôme et une qualification pour un emploi meilleur. De l'autre côté, le retour aux études est plus fréquent chez les jeunes qui étaient auparavant inscrits dans un programme universitaire ou pré-universitaire. Parmi 
eux, ceux qui sont sortis diplômés ont eu davantage tendance à s'inscrire à nouveau dans un programme universitaire ou pré-universitaire. En revanche, ceux qui sont sortis sans diplôme s'orientent aussi bien dans un programme universitaire que dans un programme professionnel. Ces retours prennent donc deux sens différents du point de vue des biographies éducatives. D’une part, les diplômés poursuivent leurs études, les étudiants inscrits dans les programmes pré-universitaires poursuivent leurs études à l'université après une pause et les diplômés du premier cycle universitaire poursuivent en études supérieures. D'autre part, les jeunes non diplômés se retrouvent plutôt dans une situation de reprise de leur scolarité. Les réorientations à la suite d'un échec scolaire ou d'un désenchantement scolaire sont emblématiques de cette situation.

Le risque de retour est par ailleurs influencé par quatre facteurs :

- Le premier facteur relève de l'entrée dans la vie adulte. Les effets de plusieurs variables comme l'âge, la situation matrimoniale, les caractéristiques de l'emploi peuvent s'interpréter à la lumière du passage vers la condition d'adulte. Ainsi, peu importe que l'on ait quitté avec ou sans diplôme et peu importe aussi le niveau d'études en jeu, plus les étudiants sont porteurs des caractéristiques attribuées à l'âge adulte, moins les retours sont fréquents. Moins les étudiants sont porteurs de ces mêmes caractéristiques, plus les retours aux études sont probables. Le retour aux études est difficilement justifiable lorsqu'on dispose déjà des avantages de la vie adulte auxquels les études sont censées donner accès. Le temps des études paraît révolu lorsqu'on a un emploi stable à temps plein, un salaire relativement élevé et un poste qualifié (Marcus, 1986). Ces résultats s'inscrivent bien dans le sens d'une désynchronisation des différents passages vers l'âge adulte avec une certaine alternance entre des périodes d'études et des périodes d'absence d'études (Galland, 1996, 2001). À cet égard, notons que ce schéma est loin de forcément correspondre au projet d'éducation et de formation tout au long de la vie. Il s'inscrit tantôt dans une logique de réorientation scolaire et professionnelle, tantôt dans l'expérimentation d'autres types d'activités, sur une courte période. De plus, le fait que plus les acteurs portent les attributs de l'âge adulte moins les retours se réalisent souligne bien que l'articulation entre les sphères d'activité et les rythmes de vie reste relativement classique.

La base de données utilisée ne permet pas cependant de préciser les motifs et les conditions sociales favorables aux reprises d'études des acteurs sociaux plus âgés. On ne peut pas savoir si l'arrêt des études suite à l'entrée dans la vie adulte constitue une pause pour se consacrer à son insertion professionnelle ou pour fonder une famille ou si c'est un arrêt définitif. L'exemple des ingénieurs est intéressant. Une fois leurs études terminées, ces derniers s'insèrent professionnellement, peuvent suivre des formations dans le cadre de leur emploi et progresser dans leur carrière. Toutefois, après quelques années, ils retourneront aux études pour obtenir un diplôme supplémentaire, très souvent en gestion (MBA), et amorcer une bifurcation de carrière vers des postes de gestionnaires ou de cadres (CEFI, 2005). 
- Le deuxième facteur est associé aux différences territoriales. Deux régions se démarquent: le Québec et les Prairies. En fait, derrière les facteurs géographiques, nous retrouvons un effet de la structuration du système de formation et un effet de la situation économique. Cette distinction souligne bien le caractère non univoque de l'usage de cette dimension dans l'analyse. Cela appelle à l'usage de variables plus précises pour distinguer les effets de l'organisation des systèmes éducatifs des impacts du contexte économique. Toutefois, dans les deux cas, il s'agit bien d'éléments analytiques d'ordre contextuel.

L'effet "Québec » tient d'abord à l'organisation spécifique de son enseignement postsecondaire. Contrairement à ce qui se pratique dans le reste du Canada, au Québec, on ne s'inscrit pas à l'université en sortant de l'école secondaire. On termine le secondaire après onze années d'études plutôt que douze, puis on suit un programme pré-universitaire de deux ans qui se conclut par un diplôme spécifique ; on entreprend ensuite le premier cycle universitaire qui dure trois ans plutôt que quatre comme dans le reste du Canada. Au cours de ce cycle d'études, le processus d'orientation scolaire et professionnelle se poursuit. De nombreux étudiants découvrent de nouveaux horizons ou connaissent des difficultés scolaires qui les incitent à changer de voie. La pause dans les études peut servir à préciser cette dernière. Autrement dit, l'effet de résider au Québec tient aussi à ce que, dans cette province, on entre à l'université après avoir déjà obtenu un premier diplôme d'études postsecondaires. L'obtention de ce diplôme peut être l'occasion de faire une pause avant d'entreprendre les études universitaires. Les universités québécoises ont aussi développé un éventail de programmes qui correspondent à une année d'études à temps plein. Cette formule est très populaire auprès des adultes de nombreuses professions et est plus utilisée au Québec que dans les autres provinces. Enfin, de nombreux étudiants ayant terminé leurs études dans l'enseignement technique décident de poursuivre leurs études à l'université après une période d'activité. Ainsi, l'effet "géographique » révèle la flexibilité du système éducatif québécois qui résulte d'une offre de formation diversifiée et des règles souples favorisant les poursuites et les bifurcations.

La situation dans la région des Prairies souligne plutôt les effets de contexte économique. Elle regroupe trois provinces du Canada : l'Alberta, la Saskatchewan et le Manitoba. Depuis plusieurs années, l'Alberta et la Saskatchewan jouissent de croissances économique et démographique fortes, soutenues principalement par le développement de l'exploitation de leurs gisements de sables bitumineux. Par ailleurs, les jeunes des Prairies sont, au Canada, proportionnellement plus nombreux à mettre fin à leurs études après le secondaire (Doray et al., 2009). Ce phénomène s'explique généralement par l'attrait du marché du travail qui offre des emplois bien rémunérés n'exigeant pas de formation longue. Cependant, nos résultats sont plus nuancés : résider dans les Prairies réduit en effet le risque de retourner aux études dans la plupart des cas, mais augmente le risque de reprendre des études dans un programme professionnel lorsqu'on détient déjà un diplôme postsecondaire. Les reprises d'études seraient plus étroitement articulées à la situation économique et elles sont sans doute favorisées par les politiques de gestion du personnel et les politiques publiques avec des objectifs de perfectionnement professionnel et de promotion. 
- Le troisième facteur relève de la logique de reproduction sociale. L'effet du capital scolaire des parents (ceux qui ont suivi des études universitaires) s'inscrit clairement dans cette logique. Cette influence positive du capital scolaire des parents corrobore ainsi l'idée selon laquelle le retour aux études est moins probable chez les groupes socialement défavorisés et les plus disposés à l'interruption des études (Marcus, 1986) ; son effet sur la démocratisation scolaire est donc d'une portée limitée. Alors que les individus issus des catégories sociales défavorisées retournent moins aux études, ceux qui sont issus des catégories favorisées y retournent plus et apparemment pour se hisser au moins au même niveau que leurs parents dans la hiérarchie de l'éducation. En d'autres termes, la reproduction sociale s'opère par le jeu de l'exclusion ou de l'auto-exclusion. Dans le cas du retour aux études, il y a apparemment un effet de rappel (Thélot, 1982) : ce que l'on n'a pas atteint à l'issue du parcours continu, on le retrouve au terme d'un parcours discontinu.

- Le quatrième et dernier facteur est lié à la mobilisation scolaire de groupes ou de catégories sociales, souvent associée à des processus de mobilisation sociale. Dans la présente analyse, la mobilisation féministe se manifeste en creux par l'absence de différence significative entre les hommes et les femmes. Du point de vue des retours aux études, les femmes comme les hommes se situent globalement sur un même pied d'égalité. Il faut y voir le résultat du mouvement des femmes qui ont investi l'école, dès que cette dernière s'est ouverte à elles, dans les mêmes conditions que celles qui prévalent pour les hommes.

\section{Conclusion}

Un des principes directeurs de l'éducation tout au long de la vie consiste à décloisonner l'accès aux études et à faciliter l'accès à l'école à tous les âges en permettant des retours aux études après des interruptions. En ce sens, il y a bien volonté de lutter contre les ségrégations fondées sur les différences d'âge.

D'une part, les retours aux études sont devenus une réalité pour nombre de jeunes qui avaient quitté les études et y sont effectivement revenus. Il y a bien ouverture du système qui permettrait de se réorienter et ou de compléter des études après les arrêts de courte durée. Toutefois, l'intensité du retour aux études diminue avec la durée de l'interruption. Plus les études s'éloignent, moins on y retourne. Plus les jeunes vieillissent, plus les chances de retourner sont faibles. Les retours sont donc largement associés aux cycles de vie et particulièrement à l'entrée dans la vie adulte. En effet, plus les individus sont porteurs des attributs de la condition adulte, moins les retours sont probables. Nous ne pouvons donc pas conclure à un décloisonnement complet selon les âges de la vie; dès lors, les retours aux études, au moins des jeunes adultes, n’apparaissent pas comme un facteur d'institutionnalisation de l'éducation tout au long de la vie.

La logique de la reproduction sociale constitue une autre source de ségrégation scolaire : le capital scolaire des parents a un effet positif sur la probabilité de retourner aux études. 
Ainsi, le processus qui régit le retour aux études relève également de la logique du cycle de vie et de celle de la reproduction sociale. Toutefois, il convient de pondérer cette conclusion. D'une part, il est possible de dépasser cela, comme l'indique la situation des femmes qui ne se distingue pas de celle des hommes et que l'on peut associer à la mobilisation scolaire des femmes. De plus, notre analyse ne porte que sur les jeunes adultes - âgés de 26 ans ou moins - et nous n'avons pas étudié les adultes plus âgés dont les conditions de vie, peut-être meilleures ou plus stables, pourraient favoriser le retour aux études ; dès lors, nous ignorons si les dimensions sociales impliquées dans les retours aux études sont aussi à l'œuvre pour les individus des générations précédentes. Ainsi, Kamanzi et al. (2009) ont montré que $45 \%$ des étudiants qui fréquentent le réseau de l'Université du Québec sont âgés de 26 ans ou plus.

Notre analyse plaide pour un examen plus approfondi du profil des étudiants qui retournent aux études, de leurs motivations, des contraintes auxquelles ils sont susceptibles d'être confrontés. Il s'agit de concevoir des politiques mieux adaptées favorisant aussi bien le retour aux études lui-même que la persévérance scolaire. Comme le notent MacGregor et Ryan (2011), ces politiques doivent concerner à la fois l'organisation pédagogique et les contenus d'enseignement, l'accès aux ressources éducatives et l'organisation institutionnelle. Notre étude s'est cependant limitée à dégager un portrait général de la situation, sans qu'il soit possible de comprendre comment les personnes construisent et mettent en œuvre leur projet de retour aux études, ainsi que les motivations à l'origine de ce projet. Pour combler cette lacune, des études qualitatives s'avèreraient utiles. Elles pourraient également s'intéresser à l'expérience scolaire de ces étudiants adultes pour appréhender leurs difficultés et la nature des soutiens dont ils ont besoin ; et ce d'autant plus qu'ils ont tendance à se comporter différemment des étudiants traditionnels face à l'apprentissage et sont vulnérables en matière de persévérance (MacGregor et Ryan, 2011). Néanmoins, les résultats obtenus nous semblent assez intéressants pour la compréhension de la question du retour aux études sur le plan théorique et l'élaboration des politiques en lien avec l'éducation tout au long de la vie.

\section{Bibliographie}

Allison P. D. (1982), "Discrete-time methods for the analysis of event histories", in S. Leinhardt, Sociological Methodology, San Francisco CA, Jossey-Bass Publishers, pp. 61-98.

Anatrella T. (1988), Interminables adolescences, les 12-30 ans, Paris, Éditions du Cerf.

ARNETT J.J. (2004), Emerging adulthood: The winding road from late teens through the twenties, Oxford and New York, Oxford University Press.

Barton F. et Podevin G. (1991), "Vingt ans de formation professionnelle continue : de la promotion sociale à la gestion de l'emploi ", Formation Emploi, 34, pp. 14-30. 
Bélanger P., Doray P. et Levesque M. (2004), La participation des femmes à la formation des adultes : une situation en changement? Quebec et Montreal, ministère de l'Emploi, de la Solidarité sociale et de la Famille et Centre interuniversitaire de recherche sur la science et la technologie.

Box-Steffensmeier J. M., Bradford S. J. (2004), Event history modeling: A guide for social scientists, Cambridge UK, Cambridge University Press.

Broekemer G.M. (2002), "A comparison of two-year and adult students. Motivations attend college and the importance of choice criteria", Journal of Marketing for Higher Education, vol. ${ }^{\circ} 12, \mathrm{n}^{\circ} 2$, pp. 31-48.

Brzinsky-Fay C., Kohler U. \& Luniak M. (2006), “Sequence analysis with Stata”, Stata Journal (4), pp. 435-460.

Centre d'Études sur les formations d'ingénieur (CEFI) (2005), Les étapes de carrière des ingénieurs, (http://.cefi.org/FEMPLOIS/FCAREERS.HTM)

Côté J.E. (2006), “Emerging adulthood as an institutionalized moratorium: Risks and benefits to identify formation", In ARnetT J.J. et TANnER J.L. (eds.), Emerging adults in America: Coming of age in the 21st century, Washington D.C., American Psychological Association Press.

Coulter X., Mandell A. (2012), "Adult education : are we moving in the wrong direction?" The Journal of Continuing Higher Education, Vol. 60, pp. 40-42.

Cox E.M., Ebbers L.E. (2010), "Exploring the persistence of adult women at Midwest Community College", Community College Journal of Research and Practice, vol. 34, pp. 337-359.

Cвоок D.B. (1997), “ The effects of adults role configurations on re-enrollment in college" , communication présentée au colloque annuel de l'American Educational Research Association, Chicago, 24-28 mars.

Davey J. A., Jamieson A. (2003), "Against the Odds: Pathways of Early School Leavers into University Education: Evidence from England and New Zealand", International Journal of Lifelong Education 22 (3), pp. 266-80.

DAYTON E. (2005), "Factors that influence adult success at community college", The Community College Enterprise, vol. 1, n 1, pp. 45-60.

DE Montlibert C. (1977), "L'éducation permanente et la promotion des classe moyennes ", Sociologie du travail vol 19, n 3, pp. 243-265.

Delors J. et alii. (1999), L'Education : un trésor est caché dedans, rapport à l'UNESCO de la Commission internationale sur l'éducation pour le vingt et unième siècle, Paris, UNESCO. 
Delors J. (1996), L'éducation : un trésor est caché dedans, Paris, Commission internationale sur l'éducation pour le vingt et unième siècle, UNESCO, 311.

Desjardins R., Rubenson K. et Milana M. (2007), Accès inégal à la formation pour adultes : perspectives internationales, Paris, UNESCO, Institut international de planification de l'éducation.

Doray P. et alii (2009), Parcours scolaires et modes de transition dans l'enseignement postsecondaire canadien, Montréal, Centre interuniversitaire de recherche sur la science et la technologie, Projet Transitions, Note de recherche 4. http://www.cirst.uqam.cal Portals/0/docs/projet transitions/TransitionsNote4-fr-Final.pdf

Doray P. (2011), « De la condition étudiante aux parcours des étudiants : quelques balises théoriques " dans PICARD F. et Masdonati J., Les parcours scolaires et d'insertion des jeunes, Québec, Presses de l'Université Laval.

Doray P., Bélanger P., et Motte A. (2005), Les facteurs de variation de la participation à l'éducation des adultes au Québec et au Canada en 1997, note de recherche CIRSTCIRDEP-MESSF, Montréal et Québec.

FAure E. et alii. (1972), Apprendre à être, Paris, UNESCO, Fayard.

Fisher D., Rubenson K. Bernatchez J., Clift R., Jones G., Lee J., MacIvor M., Meredith J., Shanahan T. et Trottier C. (2007), Canadian Federal Policies and Potstsecondary Education. Vancouver: The Center for Policy Studies in Higher Education and Training, $191 \mathrm{p}$.

Fishe D., Rubenson K., Jones G., \& Shanahan T. (2009), "The political economy of post-secondary education: A comparison of British Columbia, Ontario and Québec”, Higher Education, 5, pp. 549-566.

Galland O. (1996), "L'entrée dans la vie adulte en France. Bilan et perspectives sociologique ", Sociologie et sociétés, 28, 1, pp. 37-46.

Galland O. (2001), "Adolescence, post-adolescence, jeunesse. Retour sur quelques interprétations ", Revue française de sociologie, 42, pp. 611-640.

Gaudet S. (2007), Emerging adulthood: A new stage in the life course, Ottawa, Policy Research Initiative.

Goldberg B.J. (1985), Perceived Effects of a Financial Aid Scholarship on Returning Women Students, Research Report $\mathrm{n}^{\circ}$ 7-85, University of Maryland, $9 \mathrm{p}$.

Hango D. (2007), "Faire une pause entre les études secondaires et les études postsecondaires : déterminants et premiers résultats sur le marché du travail”, Questions d'éducation, vol..$^{\circ}$, $\mathrm{n}^{\circ} 5 \mathrm{http}: / /$ www.statcan.ca/francais/freepub/81-004-XIF/2007005/ article/10501-fr.htm 
Hango D. (2011), Différer les études postsecondaires : qui le fait et pendant combien de temps ?, Ottawa, Statistique Canada, direction du Tourisme et Centre de la statistique de l'éducation, $n^{\circ} 81-595-\mathrm{M}$;

Kamanzi P.C. et alii (coll.) (2009), Les étudiants canadiens de première génération à l'université : la persévérance aux études, Montréal : Université du Québec à Montréal, CIRST.

KeIFer N. (1990), "Econometric methods for grouped duration data" , in Hartog J., Ridder G. et Theeuwes J., Panel Data and Labour Market Studies, New York, North Holland, pp. ${ }^{\circ}$ 97-117.

Kiefer D.M. et Johnson J.A. (1997), “ Diffusing the adult student's motivation to disengage from a community College's admission process", Continuing Higher Education Review, vol. ${ }^{\circ}$ 61, pp. 104-113.

Knighton T., Hujaleh F., Iacomapo J. et Werkneh G. (2009), L'apprentissage à vie chez les Canadiens de 18 à 64 ans: premiers résultats de l'Enquête sur l'accès et le soutien à l'éducation et à la formation de 2008, Ottawa, Statistique Canada, nº 81-585M.

Kwong T. M., Mok Y. F. and Kwong Y. L. (1997), "Social factors and adult learners'motivations in re-entering higher education", International Journal of Lifelong Education, vol. ${ }^{\circ} 16, \mathrm{n}^{\circ}$ 6, pp. 518-534.

Lambert M., Zeman K., Allen M. and Bussière P. (2004), Who pursues postsecondary education, who leaves and why: Results from the Youth in Transition Survey, Ottawa, Statistics Canada.

Laplante B., María C.S., P.C. Kamanzi, P. Doray, S. Moulin (2010), Les retours aux études postsecondaires chez les jeunes adultes canadiens : une décision fortement ancrée au passé social et scolaire de l'individu (Projet Transitions, Note de recherche 12), Montréal, CIRST.

MacGregor C., Ryan T.G. (2011), "Secondary level re-entry of young Canadian adult learners", Australian Journal of Adult Learning, Vol. ${ }^{\circ}$ 51, n 1, pp. 143-160.

Marcus R. D. (1986), "Earnings and the decision to return to school”, Economics of Education Review, vol. ${ }^{\circ}$ 5, n 3, pp. 309-317.

Picard F., Trottier C., Doray P. (2011), «Conceptualiser les parcours à l'enseignement supérieur ", L'orientation scolaire et professionnelle, vol. ${ }^{\circ} 40, \mathrm{n}^{\circ} 3$, pp. 343-365.

RaYmond M. (2008), High school dropouts returning to school, Statistics Canada Catalogue $\mathrm{n}^{\circ 0}$ 81-595-M. En ligne: http://www.statcan.gc.ca/pub/81-595-m/81-595-m2008055eng.pdf

RHDSC (2006), Introduction de l'édition 2001 de la Classification nationale des professions (CNP), Ottawa, Gouvernement du Canada, Ressources humaines et Développement des compétences au Canada. 
Sftor N.S. and Turner S.E. (2002), "Back to school. Federal student aid policy and adult college enrollment”, Journal of Human Resources, vol. 37, n 2, pp. 336-352.

Smart J.C. and Pascarella E.T. (1987), "Influences on the intention to reenter higher education", The Journal of Higher Education, vol. ${ }^{\circ}$ 58, n 3, pp. 306-322.

Statistique Canada (2007), Enquête auprès des jeunes en transition, (EJET). Cohorte B, 24 à 26 ans. Guide de l'utilisateur, Cycle 4, Ottawa, Statistique Canada.

TнÉLот C. (1982), Tel père, tel fils? Position sociale et origine familiale, Paris, Dunod.

Thomas W. (2001), “The decision to return to full-time education”, Education Economics, vol. ${ }^{\circ} 9, \mathrm{n}^{\circ} 1$, pp. 37-51.

Toмкоwicz J., Bushniк T. (2003), Qui poursuit des études postsecondaires et à quel moment : Parcours choisis par les jeunes de 20 ans, Éducation, compétence et apprentissage (Document de recherche), Ottawa : Statistique Canada (Divisions Culture, tourisme et centre de la statistique de l'éducation). [81-595-MIF2003006]. 36 p. 